\title{
Age-dependent changes of microcirculation in schizophrenic patients
}

A. Seeck, Department of Medical Engineering and Biotechnology, University of Applied Sciences, Jena, Germany, Andrea.Seeck@fh-jena.de

K.J. Bär, Department of Psychiatry and Psychotherapy, University Hospital, Jena, Germany,

KARL-JUERGEN.Baer@med.uni-jena.de

A. Voss, Department of Medical Engineering and Biotechnology, University of Applied Sciences, Jena, Germany, Andreas.Voss@fh-jena.de

\section{Introduction}

The objective of this study was to investigate if age dependent changes of microcirculation in schizophrenic patients exist. Therefore, a post-occlusive reactive hyperaemia test was performed to assess dynamic changes of microcirculation in schizophrenic patients and healthy controls. Segmented spectral analysis (SSA) of the hyperaemic stage was applied to disclose more detailed information about the dynamic behaviour of blood flow in comparison with traditional total spectral analysis (TSA).

\section{Methods}

37 healthy subjects and 47 patients were enrolled and separated into two age groups of $\leq 30$ years ( 19 controls, 25 patients) and $>30$ years (18 controls, 22 patients). Spectral analysis was performed on two laser Doppler flowmetry (LDF) signals (depth of 2 and $6 \mathrm{~mm}$ ) and features in five frequency subintervals were calculated referring to endothelial (0.01$0.02 \mathrm{~Hz})$, sympathetic $(0.02-0.06 \mathrm{~Hz})$, myogenic $(0.06-0.2 \mathrm{~Hz})$, respiratory $(0.2-0.6 \mathrm{~Hz})$ and cardiac $(0.6-1.6 \mathrm{~Hz})$ activity. Short time Fourier transform spectra of blood flow in hyperaemic stage were analyzed over the whole signal applying TSA. In addition, these spectra were divided into temporal segments for applying SSA.

\section{Results}

Our results indicate significant modifications of blood flow between controls and patients more dominant in the age group $>30$ years. SSA of blood flow in this age group revealed differences in all three frequency intervals referring to local vasomotion (endothelial $\mathrm{p}=0.004$, sympathetic $\mathrm{p}=0.001$, myogenic $\mathrm{p}=0.001)$ as well as in the $\operatorname{cardiac}(\mathrm{p}=0.002)$ component whereas in the younger age group $\leq 30$ years only the cardiac $(p=0.004)$ interval was altered. TSA solely revealed significant differences in the cardiac interval for both age groups.

\section{Conclusion}

It was shown that microcirculation in schizophrenic patients is differently affected in the younger and the older age group. Only with the introduced method of SSA we were able to reveal impairments of the endothelial, the sympathetic and the myogenic mechanisms in the older age group. 